\title{
Precursors of a Mott insulator in modulated quantum wires
}

\author{
Arkadi A. Odintsov \\ Department of Applied Physics, Delft University of Technology, 2628 CJ Delft, The Netherlands; \\ Nuclear Physics Institute, Moscow State University, Moscow 119899 GSP, Russia; \\ and NTT Basic Research Laboratories, 3-1 Morinosato-Wakamiya, Atsugi-shi 243-01, Japan \\ Yasuhiro Tokura and Seigo Tarucha \\ NTT Basic Research Laboratories, 3-1 Morinosato-Wakamiya, Atsugi-shi 243-01, Japan
}

(Received 21 July 1997)

\begin{abstract}
We investigate the transport of interacting electrons through single-mode quantum wires whose parameters are periodically modulated on the scale of the electronic Fermi wavelength. The Umklapp scattering and backscattering of electrons can be described in terms of nonuniform quantum sine-Gordon-like models that also incorporate the effects of electronic reservoirs (electrodes) adiabatically coupled to the wire. We concentrate on weak Umklapp scattering and analyze the precursors of the Mott transition. At half-filling the temperature dependence of the extra resistance $\Delta R=R-\pi \hbar / e^{2}$ of a modulated quantum wire of length $L$ changes from the interaction-dependent "bulk" power-law $\Delta R \propto T^{4 K_{\rho}^{-3}}$ at high temperatures $\left(T \gg v_{\rho} / L\right)$ to the universal $\Delta R \propto T^{2}$ behavior at low temperatures $\left(T \ll v_{\rho} / L\right)$. Away from half-filling the "bulk" results are qualitatively incorrect even at high temperatures $v_{\rho} / L \ll T \ll T^{*}$ despite the fact that the electron coherence in the wire is absent in this regime. [S0163-1829(97)53144-4]
\end{abstract}

Since its discovery in 1949 the Mott transition (MT) (Ref. 1) remains the focus of intensive investigations. Much progress has been achieved in the theory of the MT in one spatial dimension (see reviews 2-4). Recent breakthroughs in technology have opened at least two intriguing opportunities for experimental observation of the MT in artificially fabricated and naturally grown one-dimensional (1D) conductors.

First, it became possible to fabricate long and clean quantum wires. ${ }^{5,6}$ This system has been successfully used for the detection of non-Fermi-liquid behavior of interacting electrons in one dimension. ${ }^{5,6}$ It should be technologically possible to modulate the electrostatic potential along the wire with the period $a=2 \pi / G$ of the order of the electronic Fermi wavelength using selective wet etching of the donor layer. By varying the concentration of electrons with an additional gate one can study the effects of the electron backscattering and Umklapp scattering on transport through modulated quantum wire (MQW). In an infinite system the electron backscattering leads to the opening of the gap at the boundary of the Brillouin zone, $G=2 k_{F}$. More interestingly, at half-filling, $G=4 k_{F}$, the Mott gap is formed ${ }^{7}$ due to the Umklapp scattering which occurs only in the interacting systems. ${ }^{2-4}$ By changing the position of the Fermi level with respect to the gaps one can effectively control electronic transport.

Second, drastic progress has been recently achieved in the synthesis of single-wall carbon nanotubes. ${ }^{8}$ Coherent electron transport and single-electron effects in this system have been demonstrated in recent experiments. ${ }^{9}$ Theoretically, low-energy properties of "armchair" nanotubes can be described by a two-chain Hubbard model at half-filling. ${ }^{10}$ The Umklapp scattering causes these (otherwise metallic) nanotubes to experience a MT at low temperatures. ${ }^{11,10}$
Previous research has addressed the effect of Umklapp scattering on the transport in uniform (and formally infinite) 1D systems. ${ }^{14,11,10}$

In a realistic experimental layout the system (MQW or carbon nanotube) is connected to external electric contacts that can be considered as reservoirs of noninteracting electrons. The presence of the reservoirs makes the system nonuniform. This has important consequences for the transport through clean ${ }^{12}$ and dirty ${ }^{13}$ quantum wires. The investigation of the effects of Umklapp scattering and backscattering of electrons on transport through realistic MQW's forms the focus of this work.

Umklapp scattering, $G \simeq 4 k_{F}$. We consider a singlechannel MQW adiabatically coupled to two perfect noninteracting 1D leads that model electronic reservoirs ${ }^{12}$ (see inset of Fig. 1). At low energies $E \ll E_{F}$ the system can be treated within the bosonization formalism., ${ }^{2,3}$ To describe the transport it is enough to consider the charge part $H=H_{\rho}+H_{U}$ of the bosonized Hamiltonian, which is decoupled from the spin part. The charge part contains the standard TomonagaLuttinger term $(\hbar=1)$,

$$
H_{\rho}=\int_{-\infty}^{\infty} \frac{d x}{\pi}\left\{\frac{v_{\rho}(x)}{K_{\rho}(x)}\left(\nabla \theta_{\rho}\right)^{2}+v_{\rho}(x) K_{\rho}(x)\left(\nabla \phi_{\rho}\right)^{2}\right\},
$$

associated with the forward scattering of electrons and the nonlinear term

$$
H_{U}=\int_{-\infty}^{\infty} d x U(x) \cos 4 \theta_{\rho}
$$

describing the Umklapp scattering. Here $\theta_{\rho}$ and $\phi_{\rho}$ are bosonic fields satisfying the commutation relation $\left[\theta_{\rho}(x), \phi_{\rho}\left(x^{\prime}\right)\right]=(i \pi / 4) \operatorname{sign}\left(x-x^{\prime}\right), v_{\rho}$ is the velocity of 


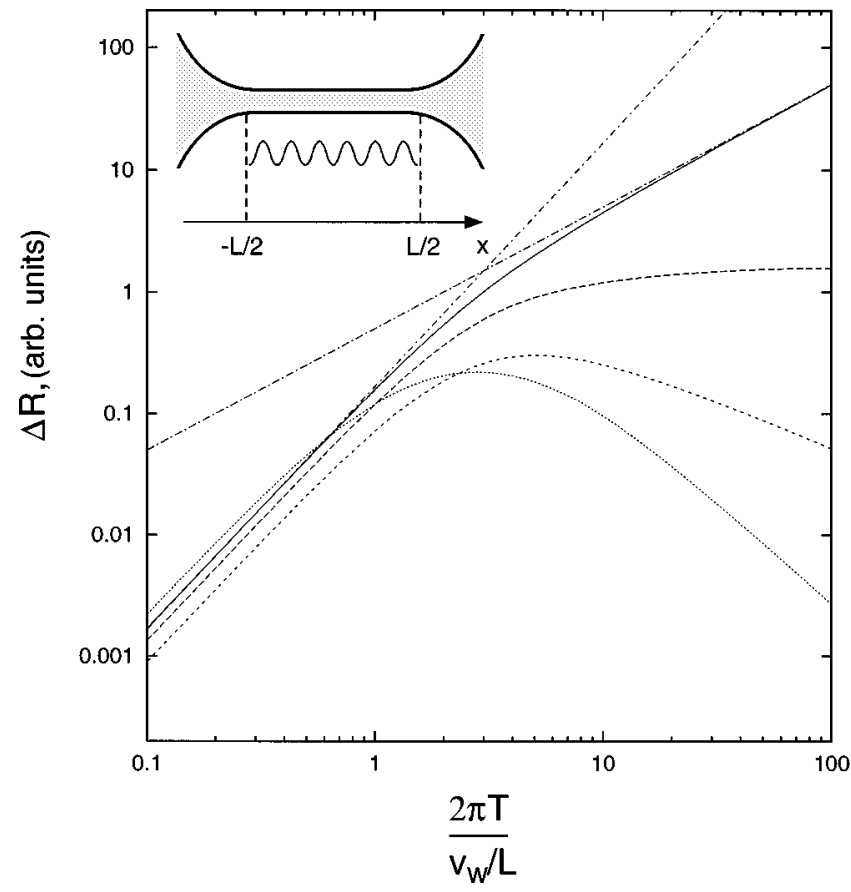

FIG. 1. The extra resistance of modulated quantum wire at halffilling $(q=0)$ as a function of temperature. $K=1,0.75,0.5,0.25$ for the curves from top to bottom at high temperatures. Dash-dotted lines correspond to the low- and high-temperature asymptotics [see text below Eq. (9)]. Inset: layout of the system.

charge excitations, and $K_{\rho}$ is a standard interaction parameter of the Tomonaga-Luttinger model $\left(K_{\rho}=1\right.$ for noninteracting electrons). The amplitude $U$ of the Umklapp scattering is proportional to the $2 k_{F}$-Fourier component $V\left(2 k_{F}\right)$ of the electron-electron interaction. In particular, for a weak periodic potential $W(x)=W_{0} \cos G x, W_{0} \ll E_{F}$ we obtain $U=V\left(2 k_{F}\right) W_{0} / 8 \pi v_{F} a$.

We will assume that the Umklapp scattering as well as the Coulomb interaction of electrons occur only in the MQW $(|x|<L / 2)$, which is characterized by position-independent parameters $\left[K_{\rho}(x), v_{\rho}(x), U(x)\right]=\left(K, v_{w}, U\right)$ in Eqs. (1) and (2). The parameters change stepwise at $x= \pm L / 2$ acquiring noninteracting values $\left(1, v_{l}, 0\right)$ in the leads $(|x|>L / 2) .{ }^{12}$ Let us note that despite the assumption that the wire is coupled to the leads via adiabatic contacts with size $R \gg 2 \pi / k_{F}$ the use of stepwise approximation is legitimate at low energies, $E \ll v_{F} / R \ll E_{F}$

To evaluate the current $I=2 e\left\langle\dot{\theta}_{\rho}\right\rangle / \pi$ through the wire we consider the Heisenberg equations for $\dot{\theta}_{\rho}$ and $\dot{\phi}_{\rho}$. The equations should be supplemented with the boundary conditions $^{16}$

$$
\left.\nabla\left\langle\theta_{\rho} \pm \phi_{\rho}\right\rangle\right|_{x \rightarrow \mp \infty}=\mu_{ \pm} / v_{l},
$$

which reflect the fact that the chemical potential $\mu_{+}\left(\mu_{-}\right)$of the left (right) reservoir determines an excess density $\rho_{+}$
( $\rho_{-}$) of rightgoing (leftgoing) electrons in the left (right) lead, $\rho_{ \pm}=\mu_{ \pm} / \pi v \quad\left(\rho_{ \pm}=0\right.$ corresponds to half-filling, $\left.G=4 k_{F}\right)$.

In what follows we will concentrate on precursors of the MT due to weak Umklapp scattering that can be treated perturbatively. We decompose the fields $\theta_{\rho}$ and $\phi_{\rho}$ into classical parts $\theta_{\mathrm{cl}}, \phi_{\mathrm{cl}}$ ( $c$ numbers) and fluctuations $\hat{\theta}, \hat{\phi}$. In the absence of Umklapp scattering $(U=0)$ the solution of the Heisenberg equations satisfying the boundary conditions (3) has the form

$$
\theta_{\mathrm{cl}}^{(0)}(x, t)=\frac{q}{4} x-\frac{e V}{2} t \quad \text { for } \quad|x|<\frac{L}{2},
$$

where $e V=\mu_{+}-\mu_{-}$is the dc voltage applied, and $q=2 K\left(\mu_{+}+\mu_{-}\right) / v_{w}=4 k_{F}-G$ characterizes a deviation of the electron density from half-filling. The current following from this solution corresponds to the Landauer formula, $I^{(0)}=\left(2 e^{2} / h\right) V$.

The correction $\Delta I$ to the current $I^{(0)}$ due to Umklapp processes arises to the second order in the scattering amplitude $U$. It can be found by expressing the difference of electronic densities $\left\langle\nabla \theta_{\rho}(-\infty)-\nabla \theta_{\rho}(\infty)\right\rangle$ at the ends of the wire from the Heisenberg equation for $\dot{\phi}_{\rho}$ and substituting the result into the boundary condition (3). After some algebra we obtain, ${ }^{17}$

$$
\begin{aligned}
\Delta I= & -2 e U^{2} \int_{-L / 2}^{L / 2} \frac{d x}{\pi} \frac{d x^{\prime}}{\pi} \int_{0}^{\infty} d t \sin \left[q\left(x-x^{\prime}\right)-\Omega t\right] \\
& \times \operatorname{Im} G\left(x, x^{\prime} ; t\right),
\end{aligned}
$$

with $\Omega=2 \mathrm{eV}$ and $G\left(x, x^{\prime} ; t\right)=\left\langle e^{4 i \hat{\theta}(x, t)} e^{-4 i \hat{\theta}\left(x^{\prime}, 0\right)}\right\rangle$, where the average is taken over equilibrium fluctuations of the field $\hat{\theta}(x, t)$ described by Eq. (1). The function $G\left(x, x^{\prime} ; t\right)$ is given by

$$
G\left(x, x^{\prime} ; t\right)=\exp \left\{-8\left\langle\hat{\theta} \hat{\theta}-2 \hat{\theta} \hat{\theta}^{\prime}+\hat{\theta}^{\prime} \hat{\theta}^{\prime}\right\rangle\right\},
$$

where $\hat{\theta}=\hat{\theta}(x, t)$ and $\hat{\theta}^{\prime}=\hat{\theta}\left(x^{\prime}, 0\right)$. The correlators (6) are related to the imaginary part of the retarded Green's function $D_{\omega}^{(R)}\left(x, x^{\prime}\right)$ via the fluctuation-dissipation theorem $\left(k_{B}=1\right)$,

$$
\left\langle\hat{\theta} \hat{\theta}^{\prime}\right\rangle=-\int \frac{d \omega}{2 \pi} e^{-i \omega t}\left(\operatorname{coth} \frac{\omega}{2 T}+1\right) \operatorname{Im} D_{\omega}^{(R)}\left(x, x^{\prime}\right) .
$$

To evaluate the retarded Green's function $D_{\omega}^{(R)}\left(x, x^{\prime}\right)$ we write down the Euclidian Lagrangian corresponding to the Hamiltonian (1), solve the Euler-Lagrange equation for the Matsubara Green's function $D_{\omega}^{(M)}\left(x, x^{\prime}\right),{ }^{12}$ and continue the latter to real frequencies, $D_{\omega}^{(R)}\left(x, x^{\prime}\right)=D_{-i \omega+\epsilon}^{(M)}\left(x, x^{\prime}\right)$, $\epsilon \rightarrow+0$. This gives the following expression for the spectral function:

$$
\operatorname{Im} D_{\omega}^{(R)}\left(x, x^{\prime}\right)=-\frac{\pi K}{4 \omega} \frac{\left(K_{+}^{4}-K_{-}^{4}\right) \cos p\left(x-x^{\prime}\right)+8 K K_{+} K_{-} \cos p\left(x+x^{\prime}\right) \cos p L}{K_{+}^{4}-2 K_{+}^{2} K_{-}^{2} \cos 2 p L+K_{-}^{4}}
$$




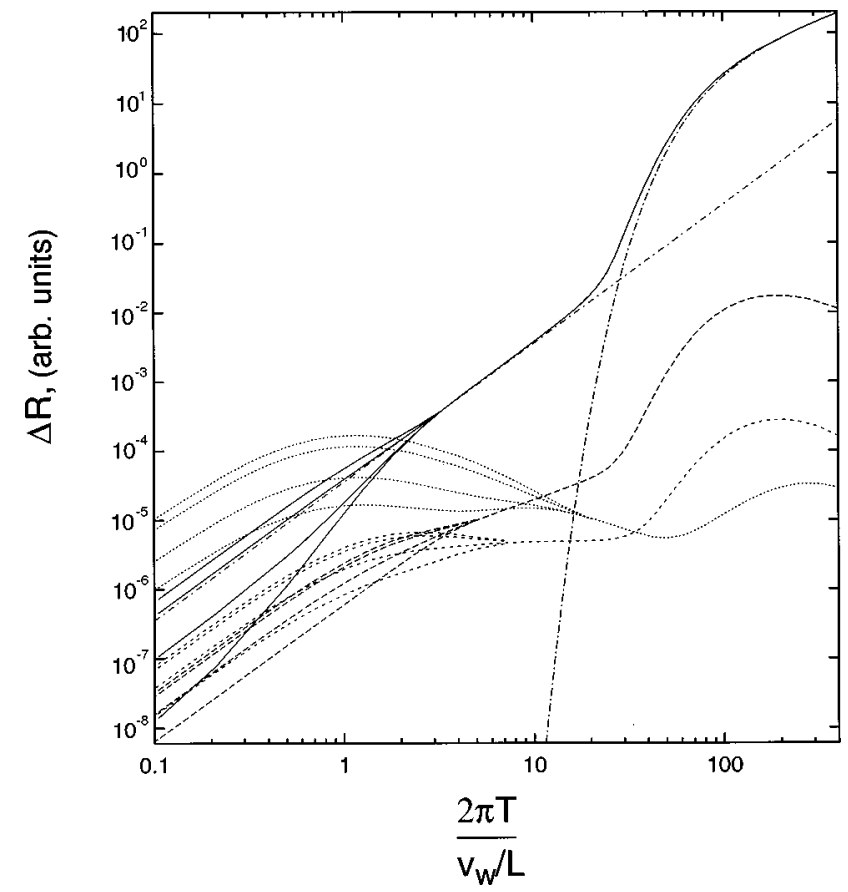

FIG. 2. The same as Fig. 1, but away from half-filling. Different curves in each family correspond to $q L / \pi=30 \ldots 31$. $K=1,0.5,0.25,0.1$ for the curves from top to bottom at high temperatures. Dash-dotted lines correspond to asymptotics at intermediate and high temperatures [see Eqs. (10) and (11) and the text in between].

where $K_{ \pm}=1 \pm K$ and $p=\omega / v_{w}$.

Although the results (5)-(8) are generally valid for nonlinear transport, in this paper we will concentrate on the linear response regime. The latter can be conveniently described in terms of the extra resistance $\Delta R=-(h /$ $\left.2 e^{2}\right)^{2} \lim _{V \rightarrow 0} \Delta I / V$ of the quantum wire due to the Umklapp scattering.

In order to elucidate the physics of the problem we start from the "noninteracting", case, $K=1$, where analytical results can readily be obtained (we imply that there is still a short-range component of the Coulomb interaction, $U$ $\left.\propto V\left(2 k_{F}\right) \neq 0\right)$. The extra resistance of MQW is given by

$$
\Delta R=R_{0} \int_{0}^{l} d \xi(l-\xi) \cos (\widetilde{q} \xi) \frac{\xi \operatorname{coth} \xi-1}{\sinh ^{2} \xi},
$$

where $R_{0}=\left(2 \pi U^{2} v_{w}^{2} / \omega_{c}^{4}\right) h / 2 e^{2}$ is the scale of resistance, $l=2 \pi T L / v_{w}$ is the dimensionless temperature, $\widetilde{q}=q v_{w} / 2 \pi T$ parametrizes the deviation from half-filling, and $\omega_{c} \sim E_{F}$ is the high-frequency cutoff in the integration (7).

We start from the case of half-filling $(q=0)$. At low temperatures $T \ll v_{w} / L$ we obtain $\Delta R=\left(2 \pi^{2} R_{0} / 3\right) L^{2} T^{2} / v_{w}^{2}$. The quadratic dependence on the length $L$ signals that the amplitudes of the Umklapp scattering sum up coherently along the MQW. At high temperatures $T \gg v_{w} / L$ the coherence is lost and the extra resistance is proportional to the length of the wire, $\Delta R=\pi R_{0} L T / v_{w}$. Note that the temperature dependence of the extra resistance changes from quadratic to linear with increasing temperature.

Away from half-filling $(q \neq 0)$ the system displays three different regimes depending on the temperature (see Fig. 2).
At low temperatures $T \ll v_{w} / L$ the interference effects show up in the oscillatory dependence of the resistance on the mismatch parameter $q$,

$$
\Delta R=\frac{4 \pi^{2} R_{0} T^{2}}{3 v_{w}^{2} q^{2}}(1-\cos q L) .
$$

In the intermediate temperature range $v_{w} / L \ll T \ll T^{*}$ (the parameter $T^{*}$ will be determined below) the oscillations disappear and the extra resistance is given by Eq. (10) with $\cos q L=0$. It might be surprising that the extra resistance does not depend on the length of the wire in the regime when the electron coherence in the wire is destroyed by thermal fluctuations.

At even higher temperatures $T \gg T^{*}$ the extra resistance shows thermally activated behavior,

$$
\Delta R=\frac{\pi R_{0} L \Delta_{q}^{2}}{2 v_{w} T} \frac{1}{\cosh \left(\Delta_{q} / T\right)-1},
$$

with $\Delta_{q}=v_{w} q / 2$, in agreement with the result for the conductance of an infinite system. ${ }^{14}$ By comparing Eq. (11) with the result for intermediate temperatures we obtain $T^{*}=2 \Delta_{q} / \ln \left[\Delta_{q} /\left(v_{w} / L\right)\right]$. Therefore, the "bulk' result (11) becomes valid only at surprisingly high temperatures.

Now we turn to the interacting case $(K \neq 1)$. The temperature dependence of the resistance is determined by the behavior of the correlator $G\left(x, x^{\prime} ; t\right)$, Eq. (6) $\left[\right.$ or $D^{(R)}\left(x, x^{\prime} ; t\right)$, Eq. (8)] at the time scale $\sim 1 / T$. At low temperatures $T \ll v_{w} / L$ this time scale corresponds to low frequencies $\omega \ll v_{w} / L$ at which the spectral function (8) is determined by noninteracting electrons in the leads, $\operatorname{Im} D_{\omega}^{(R)}\left(x, x^{\prime}\right)$ $=-\pi / 4 \omega$. For this reason, the extra resistance is proportional to $T^{2}$ as in the noninteracting case; see Figs. 1 and 2.

At high temperatures $T \gg v_{w} / L$ the behavior of the correlator $G\left(x, x^{\prime} ; t\right)$ [Eq. (6)] on the time scale $1 / T$ can be evaluated by averaging the spectral function (8) over fast oscillations with frequencies $\sim v_{w} / L$. The averaged spectral function has a simple form,

$$
\operatorname{Im} \bar{D}_{\omega}^{(R)}\left(x, x^{\prime}\right)=-\frac{\pi K}{4 \omega} \cos \left[\omega\left(x-x^{\prime}\right) / v_{w}\right]
$$

By substituting the approximation (12) into Eqs. (5)-(7) we obtain the power-law behavior of the extra resistance at high temperatures $T \gg v_{w} / L$ and half-filling $(q=0)$,

$$
\Delta R=\frac{\alpha U^{2} v_{w} L}{e^{2} \omega_{c}^{4 K}} T^{4 K-3},
$$

where $\alpha$ is a nonuniversal numerical factor (Fig. 1).

The high-temperature result (13) agrees with the lowestorder perturbative calculation of the dc conductivity of an infinite system. ${ }^{14}$ On the other hand, it is well known that in an infinite system at half-filling the Mott gap $\Delta_{M}$ is formed for an arbitrarily small amplitude $U$ of Umklapp scattering provided that the Coulomb interaction is repulsive. ${ }^{2}$ At low temperatures $T \sim \Delta_{M}$ the perturbative result ${ }^{14}$ breaks down and the resistance starts to increase exponentially. Our results are valid at arbitrarily low temperatures for sufficiently short wires, $v_{w} / L \gg \Delta_{M}$. 
Note that unlike the case of the Luttinger liquid with impurities ${ }^{13,15}$ the high-temperature behavior of the extra resistance (13) gives no direct indication of the true low-temperature properties of the system [the extra resistance (13) decreases with decreasing temperature for $K_{\rho}>3 / 4$, despite the formation of the gap]. On the other hand, the fact that the extra resistance (13) for a repulsively interacting system $\left(K_{\rho}<1\right)$ decreases somewhat slower than in the noninteracting case or even increases with decreasing temperature can be interpreted as a precursor of the Mott transition.

The temperature dependence of the extra resistance away from half-filling $(q \neq 0)$ is presented in Fig. 2. At high temperatures $T \gg \max \left(\Delta_{q}, v_{w} / L\right)$ this dependence obeys the power law (13). Note that also at intermediate temperatures $v_{w} / L \ll T \ll \Delta_{q}$ the $\Delta R(T)$ dependence is clearly affected by the interaction. In particular, for strong enough interaction the resistance shows an anomalous enhancement with decreasing temperature (see curve for $K=0.1$ in Fig. 2). This enhancement is a reminiscence of the corresponding effect at high temperatures $T \gg \Delta_{q}$. The variations of the extra resistance as a function of the mismatch parameter $q$ disappear at higher temperatures for stronger Coulomb interaction. This can be interpreted as an enhancement of quantum interference effects in the interacting system.

Electron backscattering, $G \simeq 2 k_{F}$. Apart from the Umklapp scattering (2), the backscattering of electrons is described by the Hamiltonian

$$
H_{b}=\int_{-\infty}^{\infty} d x U(x) \cos 2 \theta_{\rho} \cos 2 \theta_{\sigma},
$$

which couples the charge $(\rho)$ and spin $(\sigma)$ degrees of freedom. ${ }^{18}$ The backscattering current is given by the formula analogous to Eq. (5) with

$G\left(x, x^{\prime} ; t\right)=\exp \left\{-2 \Sigma_{j=\rho, \sigma}\left\langle\hat{\theta}_{j} \hat{\theta}_{j}-2 \hat{\theta}_{j} \hat{\theta}_{j}^{\prime}+\hat{\theta}_{j}^{\prime} \hat{\theta}_{j}^{\prime}\right\rangle\right\}$ $q=2 k_{F}-G$, and $\Omega=e V$. At filling one $\left(G=2 k_{F}\right)$ and low temperatures $T \ll v_{w} / L$ the extra resistance shows no dependence on temperature and on the interaction strengths. At high temperatures $T \gg v_{w} / L$ we obtain $\Delta R \propto T^{K_{\rho}+K_{\sigma^{-3}}}, K_{\sigma}$ being the interaction parameter in the spin channel.

In conclusion, we have analyzed the precursors of the Mott transition observable in transport through modulated quantum wires connected to electronic reservoirs. Known results for an infinite ("bulk') system are valid only at rather high temperatures $T \gg \max \left(v_{w} / L, T^{*}\right)$. In particular, at halffilling the "bulk" power-law dependence of the extra resistance, $\Delta R \propto T^{4 K-3}$ at high temperatures $T \gg v_{w} / L$ crosses over to the universal low-temperature $\left(T \ll v_{w} / L\right)$ behavior, $\Delta R \propto T^{2}$, which does not depend on the interaction in the wire. Surprisingly, away from half-filling the "bulk' results fail to be valid at the intermediate-temperature range $v_{w} / L \ll T \ll T^{*}$ despite the electron coherence in the wire is lost in this regime. An anomalous enhancement of the Umklapp scattering with decreasing temperature for strongly interacting system in this regime should be contrasted to its exponential suppression expected from the "bulk" theory. Our results are expected to be relevant also for armchair carbon nanotubes albeit the presence of the two energy bands in this system should be carefully taken into account.

We would like to thank D. V. Averin, G. E. W. Bauer, and Yu. V. Nazarov for useful discussions. We are grateful to $\mathrm{V}$. Ponomarenko for an interesting discussion at the final stage of manuscript preparation and for making us aware of the results of Ref. 19 before publication. The financial support of the European Community through HCM No. ERBCHBI-CT94-1474 and the Dutch Foundation for Fundamental Research on Matter (FOM) is gratefully acknowledged. This work was also a part of INTAS-RFBR No. 95-1305. One of us (A.O.) acknowledges the kind hospitality at the NTT Basic Research Laboratories.
${ }^{1}$ N. S. Mott, Proc. Phys. Soc. London, Sect. A 62, 416 (1949).

${ }^{2}$ J. Voit, Rep. Prog. Phys. 58, 977 (1995).

${ }^{3}$ H. J. Schulz, in Strongly Correlated Electronic Materials: The Los Alamos Symposium, 1993, edited by K. S. Bedell et al. (Addison-Wesley, New York, 1994), p. 187.

${ }^{4}$ V. J. Emery, in Highly Conducting One-dimensional Solids, edited by J. T. Devreese et al. (Plenum, New York, 1979), p. 247.

${ }^{5}$ S. Tarucha, T. Honda, and T. Saku, Solid State Commun. 94, 413 (1995).

${ }^{6}$ A. Yacoby, H. L. Stormer, N. S. Wingreen, L. N. Pfeiffer, K. W. Baldwin, and K. W. West, Phys. Rev. Lett. 77, 4612 (1996).

${ }^{7}$ Precise conditions for this are given, e.g., in Ref. 4.

${ }^{8}$ A. Thess et al., Science 273, 483 (1996).

${ }^{9}$ S. Tans, Nature (London) 386, 474 (1997).

${ }^{10}$ L. Balents and M. P. A. Fisher, Phys. Rev. B 55, 11973 (1997).

${ }^{11}$ Yu. A. Krotov, D.-H. Lee, and S. G. Louie, cond-mat/9611073 (unpublished).
${ }^{12}$ D. L. Maslov and M. Stone, Phys. Rev. B 52, R5539 (1995); V. V. Ponomarenko, ibid. 52, R8866 (1995); I. Safi and H. J. Schultz, ibid. 52, R17 040 (1995); I. Safi, ibid. 55, R7331 (1997).

${ }^{13}$ D. L. Maslov, Phys. Rev. B 52, R14 368 (1995).

${ }^{14}$ T. Giamarchi, Phys. Rev. B 44, 2905 (1991).

${ }^{15}$ M. P. A. Fisher and L. I. Glazman, cond-mat/9610037 (unpublished).

${ }^{16}$ R. Egger and H. Grabert, Phys. Rev. Lett. 77, 538 (1996).

${ }^{17} \mathrm{~A}$ similar expression has been obtained in the theory of a Coulomb drag of spinless Luttinger liquid; see D. V. Averin and Yu. V. Nazarov (unpublished).

${ }^{18}$ More detailed consideration of this case will be presented elsewhere.

19 V. V. Ponomarenko and N. Nogaosa, Phys. Rev. Lett. 79, 1714 (1997). 